\title{
REVIEWS
}

\section{Managing diabetes with nanomedicine: challenges and opportunities}

\author{
Omid Veiseh ${ }^{1-3 *}$, Benjamin C. Tang ${ }^{2,3 *}$, Kathryn A. Whitehead ${ }^{4}$, Daniel G. Anderson ${ }^{1-3,5,6}$ \\ and Robert Langer ${ }^{1-3,5,6}$
}

Abstract | Nanotechnology-based approaches hold substantial potential for improving the care of patients with diabetes. Nanoparticles are being developed as imaging contrast agents to assist in the early diagnosis of type 1 diabetes. Glucose nanosensors are being incorporated in implantable devices that enable more accurate and patient-friendly real-time tracking of blood glucose levels, and are also providing the basis for glucose-responsive nanoparticles that better mimic the body's physiological needs for insulin. Finally, nanotechnology is being used in non-invasive approaches to insulin delivery and to engineer more effective vaccine, cell and gene therapies for type 1 diabetes. Here, we analyse the current state of these approaches and discuss key issues for their translation to clinical practice.

${ }^{1}$ Department of Chemical Engineering, Massachusetts Institute of Technology. 77 Massachusetts Ave., Cambridge, Massachusetts 02139, USA.

${ }^{2}$ David $H$. Koch Institute for Integrative Cancer Research, Massachusetts Institute of Technology. 77 Massachusetts Ave., Cambridge, Massachusetts 02139, USA.

${ }^{3}$ Department of Anesthesiology, Boston Children's Hospital, 300 Longwood Ave., Boston, Massachusetts 02115, USA. ${ }^{4}$ Department of Chemical Engineering, Carnegie Mellon University, 5000 Forbes Ave. Pittsburgh, Pennsylvania 15213, USA.

${ }^{5}$ Division of Health Science and Technology,

Massachusetts Institute of Technology, Cambridge, Massachusetts 02139, USA. ${ }^{6}$ Institute for Medical Engineering and Science, Massachusetts Institute of Technology, Cambridge, Massachusetts 02139, USA.

* These authors contributed equally to this work.

Correspondence to R.L. e-mail:rlanger@mit.edu

doi:10.1038/nrd4477 Published online

28 November 2014
Diabetes mellitus is defined as a group of metabolic disorders characterized by high blood glucose levels (hyperglycaemia) ${ }^{1}$. The incidence of diabetes is rising; the number of affected patients worldwide is expected to increase from over 280 million adults today to over 400 million adults by 2030 (REF. 2). The total annual global costs associated with the treatment of diabetes and its complications amount to US $\$ 500$ billion $^{3}$, not including indirect costs associated with lost work time.

Type 1 diabetes, also known as juvenile diabetes, accounts for $10 \%$ of all diabetes mellitus cases ${ }^{4}$. It results from a deficiency in insulin - a 51-amino-acid peptide produced by the $\beta$-cells of the islets of Langerhans in the pancreas - which regulates blood glucose levels by stimulating liver and muscle cells to take up glucose from the blood ${ }^{4}$. This deficiency stems from an autoimmune response in affected individuals that leads to the T-cell-mediated destruction of $\beta$-cells and subsequent hypoinsulinaemia and hyperglycaemia ${ }^{5}$. Type 2 diabetes, unlike type 1 diabetes, is often categorized as a lifestyle disease ${ }^{6}$, and is associated with obesity and a lack of physical activity. Patients with type 2 diabetes develop insulin resistance - that is, their response to insulin produced by $\beta$-cells (for example, after a meal) is blunted, again leading to hyperglycaemia ${ }^{7}$.

Persistent glycaemic control is a key determinant of long-term outcomes for patients with diabetes ${ }^{8}$. The goal of management for both type 1 and type 2 diabetes is the maintenance of blood glucose levels within healthy normoglycaemic ranges (70-140 mg per $\mathrm{dl}$ or $4-8 \mathrm{mM}$; known as euglycaemia $)^{9}$. When left untreated, prolonged hyperglycaemia can lead to blindness, kidney and heart disease, nerve degeneration and increased susceptibility to infection ${ }^{10}$. Conversely, insulin overtreatment may cause hypoglycaemia, which can lead to seizures, unconsciousness or death ${ }^{11}$.

For type 1 diabetes patients, insulin replacement therapy is prescribed with the goal of mimicking natural fluctuations in insulin levels throughout the day ${ }^{12}$. Typical treatment includes injections of long-acting insulin (with a longer plasma half-life than regular insulin) to provide a basal level of insulin, which is supplemented with bolus injections of fast-acting insulin (with a shorter plasma half-life) at mealtimes ${ }^{12,13}$. For type 2 diabetes, initial treatment focuses on delaying disease progression through exercise and regulation of meals ${ }^{1}$. Patients also receive oral and/or injectable medication that improves insulin production and function ${ }^{6}$. However, insulin replacement therapy is often ultimately required as native insulin production diminishes .

Owing to the harsh environment of the gastrointestinal tract, insulin and other macromolecular diabetic therapies (that is, glucagon-like peptide 1) must be injected subcutaneously, which can be painful and inconvenient, leading to poor patient compliance ${ }^{14}$. Moreover, this conventional form of insulin replacement therapy is 'open loop', meaning that it relies on a historical understanding of the patient's unique blood glucose profile in response to various meals and insulin treatments to determine insulin dosages ${ }^{8}$. Several technologies have been developed to overcome the drawbacks of injection therapy by dynamically controlling insulin levels with 
Hyperglycaemia

A condition of high blood glucose levels, typically $>200 \mathrm{mg} / \mathrm{dL}$

Insulin

A peptide hormone that is produced by $\beta$-cells in the pancreas. It regulates the metabolism of carbohydrates and fats and reduces blood glucose by promoting the absorption of glucose from blood to skeletal muscles and fat tissue. real-time data, while reducing the patient burden associated with treatment (BOX 1). These technologies include both insulin pumps and continuous glucose monitors ${ }^{15}$. One notable example is the dual hormone (insulin and glucagon) bionic pancreas glycaemic control system, which was recently evaluated in a Phase II trial in patients with type 1 diabetes ${ }^{16}$. This system was shown to significantly improve glycaemic control while reducing the frequency of hypoglycaemic episodes ${ }^{16}$.

Despite these technological advances, it remains difficult to maintain ideal glucose levels using insulin replacement therapy in the vast majority of patients ${ }^{15}$ (BOX 1). A retrospective study of patients with diabetes estimated that $\sim 50 \%$ of patients do not achieve their target

\section{Box 1 | Improved technologies for insulin replacement therapy}

For patients with diabetes mellitus, traditional insulin replacement therapy can be painful and time consuming ${ }^{177}$. In addition, the lag between glucose measurement and insulin dosing, combined with delayed absorption of insulin following subcutaneous injection, limits tight blood glucose control and can lead to periods of hyperglycaemia (see the figure).

Several technologies have been developed to improve patient compliance associated with insulin replacement therapy, while also improving the dynamic control of blood glucose levels. For example, externally worn pager-sized insulin pumps have been developed that contain a replaceable depot of insulin connected to a subcutaneously implanted cannula. The pager can be programmed to deliver a basal level of insulin throughout the day as well as bolus insulin dosages on demand for meals through continuous insulin infusion. Alternatively, continuous glucose monitors (CGMs) are externally carried portable devices that provide near real-time measurements of blood glucose without the pain of repeated finger pricks ${ }^{13,178}$. Sensors are inserted subcutaneously and measure glucose levels in the interstitial fluid, which provides an estimate for blood glucose levels.

Most recently, microcomputer-controlled closed loop insulin delivery systems are being developed, where CGMs are used in conjunction with insulin pumps to automatically calculate and inject appropriate doses of insulin ${ }^{179,180}$. The goal of this technology is to provide a 'patient intervention-free' insulin replacement therapy. Here, the CGMs are linked directly, and are used in conjunction with insulin pumps to automatically calculate and inject appropriate doses of insulin ${ }^{179,180}$. Although these closed loop systems improve on glucose control, they do not achieve true euglycaemia as glucose levels are measured in the interstitial fluid and insulin is injected subcutaneously, which both present a time delay in diffusing to and from the bloodstream. Appropriate safety mechanisms are critical in closed loop systems such as these to prevent insulin overdose, which can lead to fatal hypoglycaemia.

Even with these technologies to improve patient compliance and glucose control, there are still major drawbacks. Insulin pumps and CGMs are expensive, and implanted sensors and cannulas increase the patient's risk of infection, inflammation and scarring ${ }^{8,15}$, and they also require frequent maintenance and replacement owing to the foreign body response, increasing effort and cost to patients ${ }^{8}$.

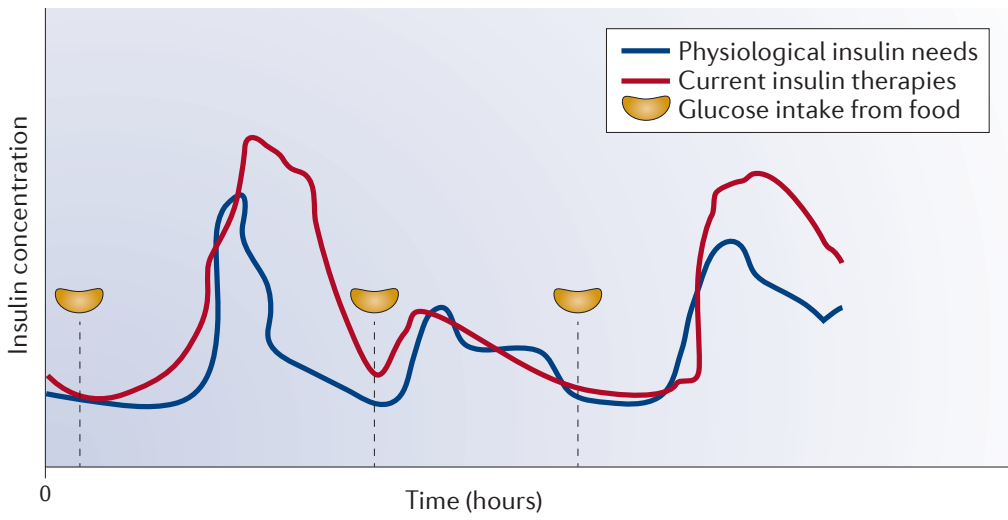

glycaemic levels throughout the day ${ }^{17}$. Contributing factors include: the open-loop nature of current therapies, whereby insulin is injected into the subcutaneous space (as opposed to the portal blood where insulin is secreted from the pancreas); approximating subcutaneous fluid as having the same glucose concentration as blood; and poor patient compliance ${ }^{1}$. To provide clinical improvements, future therapies need to be easier to use while achieving tighter glycaemic control, better safety profiles and, ideally, a reduced cost to manufacture and implement into clinical practice ${ }^{15}$. Towards these goals, scientists are working to enable alternative routes of insulin administration ${ }^{18}$, optimize insulin pharmacokinetics ${ }^{12}$ and develop new therapeutic entities ${ }^{19}$.

Over the past 20 years, nanotechnology has improved both diagnostics and therapeutics in several medical fields, including oncology and cardiology $y^{20-23}$. Indeed, nanoparticles and nanoscaled materials have many physi$\mathrm{cal}$, chemical and biological properties that render them attractive for biomedical applications $\mathrm{s}^{21,24}$. Nanoparticles are used to deliver both small-molecule and large macromolecular (that is, DNA, RNA and proteins) therapeutics, as well as to diagnose and monitor the progression of disease $e^{25}$. A myriad of novel nanoparticle formulations with varying architectures have been fabricated for biomedical applications, including liposomes, polymer nanoparticles, nanostructures, metallic nanoparticles, stimuli-responsive nanoparticles and nanofabricated devices ${ }^{26-33}$. Here, we review the developing role of nanotechnology in diabetes management ${ }^{34}$, from diagnosis and disease monitoring to therapeutics (FIG. 1). We focus on the most mature technologies in each category that we feel are most likely to have an impact on the treatment of diabetes in the near future.

\section{Diagnosis and disease monitoring}

Advances in nanotechnology, molecular imaging and biomedical imaging tools are creating new opportunities for early diagnosis, staging and monitoring of disease progression for patients with type 1 or type 2 diabetes ${ }^{35}$. Early detection of diabetes and identification of disease progression are important aspects of disease management $^{35}$. For example, as diabetes progresses, there is a reduction in $\beta$-cell mass and its respective insulin production and secretion ${ }^{36}$. Although the quantification of functional $\beta$-cells may enable physicians to prescribe more successful therapies and allow scientists to develop improved $\beta$-cell-targeted therapies, direct measurement of $\beta$-cell mass is impractical as it requires post-mortem autopsy. In recent years, opportunities to assess $\beta$-cell mass using imaging have evolved with the development of $\beta$-cell-targeting peptide dyes ${ }^{37}$ and antibody-dye conjugate ${ }^{38}$, but these are generally reserved to excised tissue samples requiring invasive procedures. Alternatively, nanoprobes are being developed with $\beta$-cell specificity and high contrast ${ }^{39}$, which may enable clinicians and researchers to non-invasively quantify in vivo endogenous $\beta$-cell mass ${ }^{40}$, survival of exogenous transplanted islets ${ }^{41}$ and the performance of islet cells in cell replacement therapy ${ }^{42-46}$. Various non-invasive imaging techniques are being investigated for the visualization of $\beta$-cell 


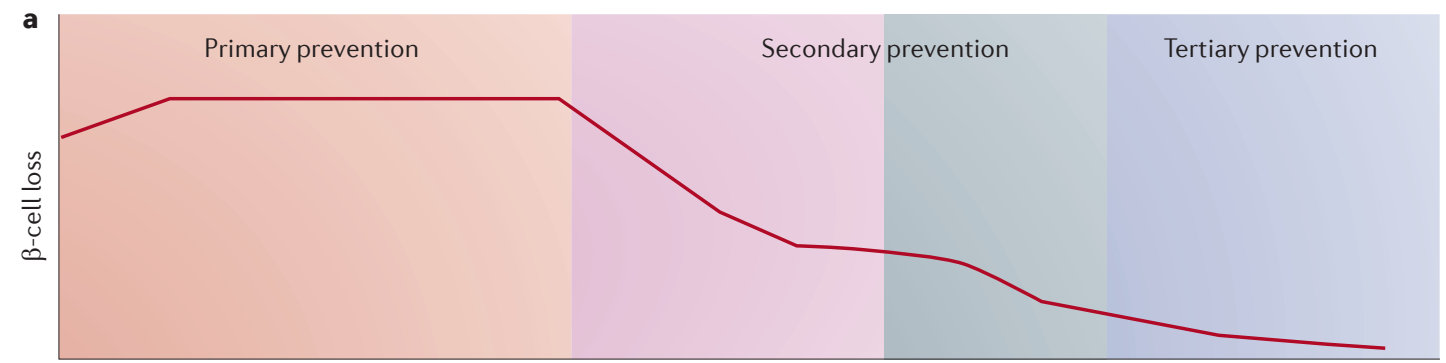

Progression of diabetes

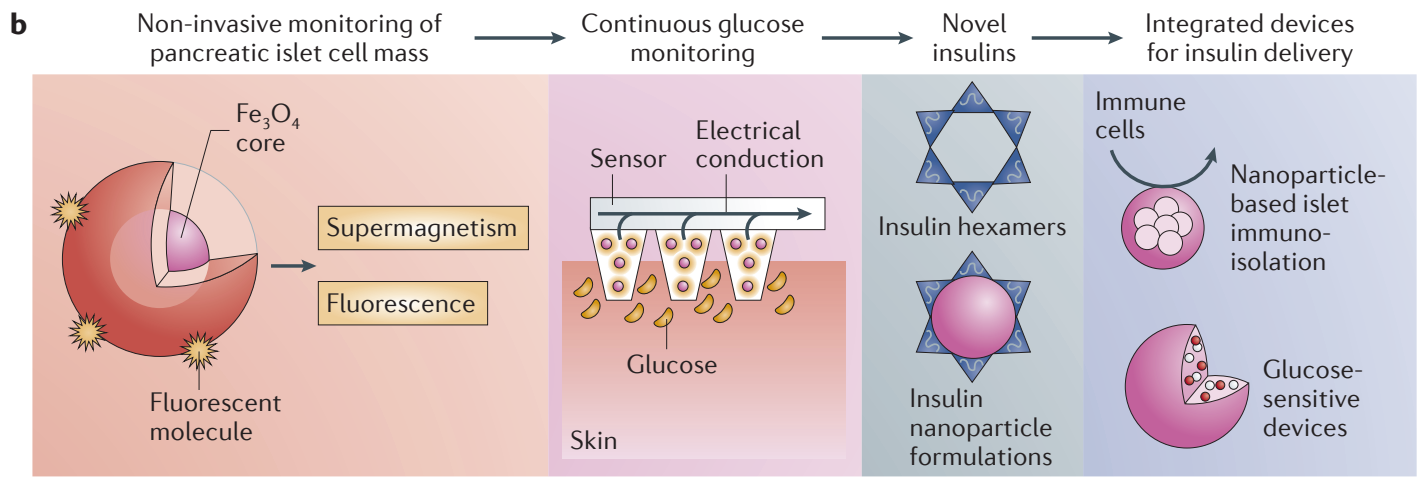

Cells in the pancreas that are located in the islets of Langerhans and that store and secrete insulin.

\section{Hypoinsulinaemia} A condition of abnormally low concentrations of insulin in the blood.

\section{Hypoglycaemia}

A condition of low blood glucose levels, typically $<70 \mathrm{mg} / \mathrm{dL}$.

\section{Glucagon}

A peptide hormone that is produced by $\alpha$-cells in the pancreas and raises blood glucose levels.

\section{Open loop}

A form of insulin replacement therapy whereby the required insulin levels are empirically estimated by blood glucose measurement and meal intake and insulin is injected by the patient at different times throughout the day.

\section{Magnetic resonance} imaging

(MRI). Imaging technique by which strong magnetic fields are applied to the area of interest, exciting hydrogen atoms to emit a radio frequency signal, which is then captured. T1 (spin-lattice) and T2 (relaxation) processes can be captured to assess different types of tissue

Inflammation

Biological response of tissues to harmful stimuli, such as

foreign objects and dead cells.

Figure 1 | Nanotechnology-based approaches to address challenges in the diagnosis and treatment of diabetes. a|The progression of diabetes results in a loss in $\beta$-cell mass, which can be subcategorized into three stages: primary, secondary and tertiary. As the disease progresses through each stage, new types of therapies are necessary to help slow advancement to the subsequent stage. $\mathbf{b} \mid$ Highlighted below the profile illustrating the progressive loss in $\beta$-cell mass are potential nanotechnology-based interventions that could be developed to address patient needs at the various stages of disease progression. A number of examples are highlighted, including: nanoparticle-based contrast agents to improve early diagnosis of the onset of type 1 diabetes; nanoparticle-based continuous glucose sensors that can facilitate frequent monitoring of blood glucose levels with improved accuracy and patient comfort; nanoparticles to improve the pharmacodynamics of insulin in order to better mimic the physiological needs of the body; and nanotechnology-based protection of transplanted pancreatic islet cells. These approaches can be used as highlighted to help maintain healthy normoglycaemic levels in patients with diabetes.

mass, including computed tomography (CT), positron emission tomography (PET) and magnetic resonance imaging $(\mathrm{MRI})^{47}$.

Several magnetic nanoparticle probes have been developed as contrast agents for $\beta$-cell imaging ${ }^{46,48}$. In particular, superparamagnetic iron oxide nanoparticles (SPIONs) are attractive in that they are biocompatible and can degrade into iron and oxygen ${ }^{31}$. The superparamagnetic properties enable these nanoparticles to be targeted using magnetism, tracked using MRI and used as magnetic triggers for drug release $\mathrm{e}^{20,49}$. SPIONs have been developed to monitor immune cell infiltration and subsequent pancreatitis as an early detection tool for diagnosing diabetes ${ }^{42}$. In a pilot clinical study, non-diabetic healthy volunteers and patients with recent-onset diabetes were infused with a clinically approved SPION-based MRI contrast-imaging agent, ferumoxtran-10 - a dextran-coated iron oxide nanoparticle that, owing to its size and surface properties, is readily taken up by macrophages - and scanned using a $1.5 \mathrm{~T}$ clinical MRI instrument to monitor pancreatitis ${ }^{50}$. The study enabled visualization of the pancreas and, more importantly, demonstrated a twofold difference in the T2 relaxation time of the pancreas in diabetic patients versus healthy volunteers owing to ongoing islet inflammation ${ }^{50}$.
The direct imaging of $\beta$-cell mass via iron oxide nanoparticles can also be used to monitor endogenous and exogenously transplanted islet cells ${ }^{45}$. Ferrimagnetic iron oxide nanocubes possess high relaxivity, which increases MRI resolution, allowing the visualization of single cells in pancreatic islets using a clinical MRI instrument ${ }^{44}$. Although major advances have been made in the development of imaging probes for monitoring inflammation and $\beta$-cell biomass, a need remains for molecularly targeted probes that can report directly on islet functionality in vivo. Specifically, the development of unique biomarkers that are specific for the $\beta$-cell surface and can monitor $\beta$-cell stress or dysfunction could help accelerate the clinical assessment of therapies to promote $\beta$-cell health and survival, as well as the stratification of patients by disease status for targeted therapies.

\section{Glucose sensors}

Frequent monitoring of blood glucose levels provides patients and physicians with an understanding of diabetes progression and the efficacy of therapies ${ }^{13}$. A number of technologies are currently available that facilitate outpatient self-administered blood glucose testing ${ }^{51}$. Unfortunately, lag times, lack of precision and difficulty with patient 


\section{Box 2 | Current glucose sensors and future goals}

Currently, most patients with diabetes depend on handheld glucometers for monitoring glycaemic levels ${ }^{181}$. These devices rely on a single sampling of blood collected through finger pricks and are typically used only a few times a day (on average four to six times a day) ${ }^{1}$. Continuous glucose monitors (CGMs), which are subcutaneously implanted amperometric sensors (typically inserted into the fatty layer of abdominal skin) that emit an electrical current in response to glucose oxidation, continually sample interstitial fluid ${ }^{51}$. Unfortunately, current versions of CGMs produce measurements that lag 5-15 minutes behind blood glucose levels owing to the diffusion of glucose from the blood to the interstitial fluid ${ }^{180}$. Additional drawbacks that have limited their widespread use include the invasive implantation procedure, the frequent need for replacement because of fouling and sensor instability, and a requirement to calibrate the sensor numerous times throughout the day using handheld glucometers ${ }^{180}$. Next-generation sensors will need to provide high accuracy for prolonged periods while being patient-friendly.

Glucose oxidase

An enzyme that catalyses the oxidation of glucose into hydrogen peroxide and D-glucono- $\delta$-lactone

Amperometric

Relating to the measurement of changes in electrical

current of an electrode with an applied voltage in response

to the presence of an analyte.

Carbon nanotubes

Allotrope of carbon that

takes a cylindrical shape.

Voltammetric

A subset of amperometry,

where the applied voltage

is additionally varied.

Phenylboronic acid

(PBA). Mild Lewis acid that

binds reversibly to 1,2-and

1,3-diols, such as glucose. use remain major challenges (BOX 2). Furthermore, recent guidelines published by the US Food and Drug Administration (FDA) indicate that more stringent accuracy and reliability requirements will be imposed in the near future for glucose sensors ${ }^{52}$. Nanotechnology has the potential to enable the development of improved sensors.

In general, sensing devices are constructed by assembling three key components: a detector that measures blood glucose concentrations; a transducer that converts measurements into output signals; and a reporter that processes the generated signal into data that can then be interpreted by the patient or physician. There are three main classes of glucose-sensing molecules that are being used to engineer nanoparticle-based glucose sensors. These include glucose oxidase, glucose-binding proteins and glucose-binding small molecules (FIG. 2a). When coupled with nanoparticles engineered as transducers, these glucose-specific detecting molecules are enabling the design of new types of sensors that have the potential to be more patient-friendly, provide rapid measurements and improve precision ${ }^{53}$ (FIG. 2b).

The first generation of glucose nanosensors utilize the amperometric glucose oxidase-sensing technology as traditional blood glucose sensors ${ }^{54}$. Glucose oxidase possesses a high level of specificity for glucose, and reacts under biological environments (that is, in blood and urine) to enzymatically convert glucose into D-glucono$\delta$-lactone (which hydrolyses into gluconic acid) and hydrogen peroxide ${ }^{55}$ (FIG. 2a). The oxidation results in an electric current that is proportional to glucose concentration $^{56}$. Nanosensors containing glucose oxidase have been built onto the surface of metallic nanoparticles, including palladium, gold and platinum nanoparticles, as well as carbon nanotubes ${ }^{57-59}$. The optimal material remains unclear as each has its unique advantages, such as stability and ease of manipulation, and its limitations, such as long-term accumulation and biocompatibility. Glucose oxidase-based approaches have one inherent disadvantage, in that glucose oxidase can have considerable batch-to-batch variability in activity, and its activity can diminish over time ${ }^{56,60}$. It also requires a constant oxygen level, $\mathrm{pH}$ and temperature, as well as frequent recalibration for a reliable readout ${ }^{55}$.
To address the limitations of glucose oxidase, nonenzymatic nanosensors have been developed to improve reliability through the elimination of drift - the continuous decrease in output signal in relation to glucose concentration caused by enzyme degradation and loss of activity - and inconsistent enzyme activity ${ }^{61-63}$. One type of non-enzymatic glucose sensors function by oxidising glucose using a metal oxide catalyst such as copper oxide ${ }^{62,64}$ or gold nanoparticles ${ }^{61}$. This amperometric approach with oxidation-based sensors involves the use of an applied voltage to drive the reaction; a battery and a device is required to measure the resulting current, making it less convenient for the patient owing to the increased size of the sensor.

Another non-enzymatic approach is based on the binding of glucose to the sensor to provide a fluorescent ${ }^{65}$ or voltammetric readout ${ }^{53}$. Transcutaneous fluorescencebased glucose sensors are currently being evaluated in clinical trials, and early data suggest that this technique can produce a reliable output for tracking blood glucose levels ${ }^{66}$. In these devices, when glucose displaces water in a binding pocket on the sensor, there is a shift in electron density that can be measured as a voltammetric or fluorescent output ${ }^{67}$. Glucose-binding moieties that have been used for such applications include natural molecules such as lectins $s^{67,68}$, synthetic molecules such as phenylboronic acid (PBA) $)^{69,70}$, and molecularly imprinted polymer hydrogels based on polyacrylamides ${ }^{63}$ and polyallylamines ${ }^{63,71}$. One of the most commonly used lectins for glucose sensing is concanavalin A (ConA), which is derived from the jack bean plant and binds specifically and reversibly to glucose $^{72}$. Another is glucose-binding protein, a bacterial surface protein that undergoes a conformational change upon binding to glucose $\mathrm{e}^{73}$. PBA is an organic molecule (FIG. 2a) that can reversibly bind to 1,2- or 1,3-cis-diols, such as glucose, to form cyclic esters ${ }^{74}$. When these moieties are associated with carbon nanotubes ${ }^{75,76}$ or nano-optodes ${ }^{77,78}$, they can potentially convert the binding event into a voltammetric output or cause a shift in fluorescence spectra. A major advantage of such devices is that they do not require a battery and may therefore function continually and for longer periods ${ }^{79}$.

Nanoparticles such as semiconducting quantum dots and single-walled carbon nanotubes are being developed as fluorescence-emitting components of such sensors ${ }^{74,79}$. In recent years, some of these formulations have been described in the literature ${ }^{75,80,81}$. Earlier versions utilized a glucose oxidase-based detector, which has a limited in vivo lifetime ${ }^{80}$. Other particles in development use sensing molecules such as PBA to improve in vivo fidelity and longevity ${ }^{75,76}$. When properly engineered, sensors based on carbon nanotubes have demonstrated remarkable longevity. For example, carbon nanotubes that were engineered as nitric oxide sensors and embedded in a biocompatible alginate gel matrix remained functional for more than 400 days when implanted subcutaneously into mice ${ }^{82}$. However, although these results are very promising, additional efforts towards improving the reliability of measurements may be needed before this approach can be translated into the clinic. Concerns remain regarding the safety of carbon nanotubes, which 
Sensors based on boronic acid derivatives<smiles>Cc1ccccc1</smiles>

Particle
$\mathrm{R}$<smiles>Cc1ccc([B-](O)(O)O)cc1</smiles>
$\mathrm{OH}$

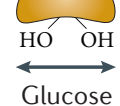

$\mathrm{R}$

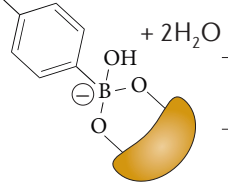

Glucose binding or release produces resonance shifts
- Rapid response rates - Long-term high fidelity

- Lack of glucose specificity

Sensors based on ConA or glucose-binding protein

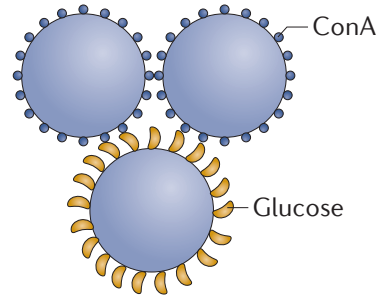

$\uparrow$ [Glucose]

binds to ConA

Nanoparticles

break apart
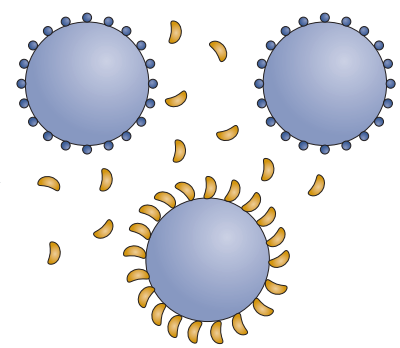

- High glucose specificity - Rapid response rates

- Susceptible to degradation

\section{b Nanoparticles as transducers}

\section{Fluorescence}

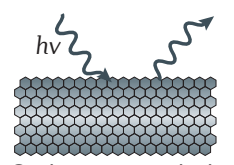

Carbon nanotubules
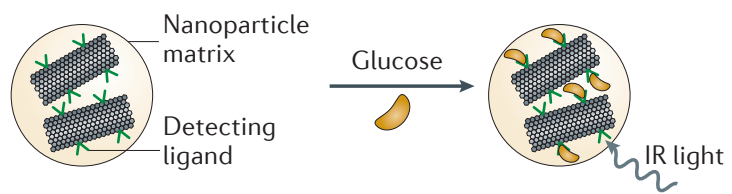

Indirect ratiometric

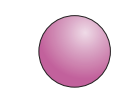

Noble metal nanoparticles

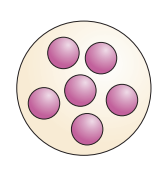

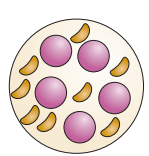

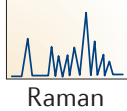

- Ultra-fast readout

- Long-term function without

battery

- Improved precision in

hypoglycaemic range

\section{Amperometric}

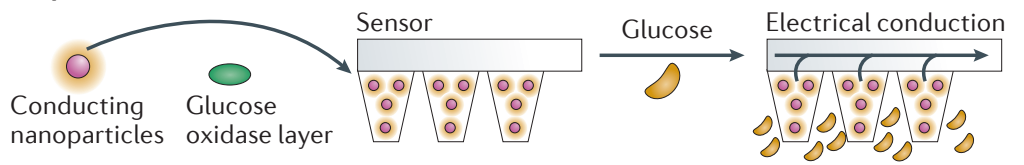

- Reliable proven technology

- Requires battery

- Frequent calibration needed

Figure 2 | Nanotechnology-based glucose sensor technologies. Sensing devices are constructed by assembling a detector that measures blood glucose concentrations and a transducer that converts measurements into output signals. a | There are three main classes of glucose-sensing molecules that are being used to engineer nanoparticle-based glucose sensors: glucose oxidase, glucose-binding proteins and glucose-binding small molecules. Highlighted next to the description of each detection molecule are the strengths (green boxes) and weaknesses (red boxes) associated with each technology. $\mathbf{b}$ | These glucose-detecting molecules can be coupled to nanoparticles engineered as transducers with unique optical or electrical properties, as well as the ability to produce surface plasmon resonance. The strengths (green boxes) and weaknesses (red boxes) associated with each technology are shown. ConA, concanavilin A; hv, excitation light; IR, infrared; PBA, phenylboronic acid. 
may be a barrier to their clinical translation ${ }^{83}$. Initial pilot studies in mice have suggested that when carbon nanotubes are properly engineered with hydrophilic and biocompatible coatings, their potential toxicity can be minimized $^{84,85}$. However, the long-term safety profile of carbon nanotubes remains unknown. Another challenge associated with fluorescence glucose nanosensors is the need for device biocompatibility as well as reliable calibration of fluorescent signals across multiple skin regions on a single patient, which can vary in colour, thickness and hair density ${ }^{86}$.

Owing to their small footprint, multiple nanosensors may be placed throughout the body to monitor blood glucose in parallel, reducing dependency on a single sensor and the risk of total sensor failure ${ }^{87}$. They may also be placed in more physiologically relevant locations, including in circulating blood or the lining of blood vessels, which would sample blood glucose directly, rather than in the subcutaneous interstitial fluid where current sensors are implanted ${ }^{53}$.

The development and testing of glucose nanosensors is at an early stage, with in vivo evaluation having only been carried out in animals so far. The non-enzymatic technologies that have been developed for nanosensors may be readily translated to and developed into nextgeneration continuous glucose-monitoring sensors with less variability than current glucose oxidase-based sensors. The non-invasive nanosensors with optical or fluorescent readouts hold the most promise to replace the current standard of manual glucose sensing based on finger pricking. Such optically based sensors have generated considerable interest because they do not require a battery or an enzyme-based catalyst and can be engineered to provide ultrafast readouts at rates that are significantly higher than current electrode-based sensors.

\section{Insulin delivery}

Nanotechnology is being used to improve the ease, efficacy and safety of insulin replacement therapy ${ }^{88,89}$. For example, long-acting nanoparticulate formulations of insulin have been developed to minimize the frequency of injections ${ }^{90}$. Since the first description of smart, glucose-responsive insulins in 1979 (REF. 91), there has been considerable interest in developing insulin formulations with activity that is dependent on glucose concentration, as this could facilitate tighter glycaemic control while minimizing the potential for hypoglycaemia ${ }^{92}$. In addition, new formulations are being explored to enable alternative, less invasive routes for insulin delivery (that is, oral $^{93}$, transdermal ${ }^{94}$ and inhaled deliver $y^{95}$ ). Multifunctional nanoparticle formulations have the potential to address many of these challenges ${ }^{96}$. Insulin-delivering nanoparticle technology is rapidly maturing, and some early-generation carriers are in the clinic $^{97}$. Here, we highlight the areas where nanotechnology has had an impact and holds substantial potential in improving the delivery of insulin.

Glucose sensor-dependent insulin delivery. As well as improving the reliability of detecting glucose levels, the sensors discussed above could be used as glucoseresponsive insulin delivery systems. These delivery
Figure 3 | Development of glucose-responsive nanoparticles for insulin delivery. a|There are several mechanisms by which glucose-sensing triggers can be integrated with nanoparticle design to facilitate glucose-responsive behaviour. Nanoparticles prepared using polymers that are molecularly imprinted with glucose and phenylboronic acid (PBA) could form supramolecular assemblies through reversible hydrogen-bonding interactions between glucose and PBA molecules. These nanoparticle assemblies would then be sensitive to glucose concentrations in their localized environment through the competitive binding of glucose from the environment to PBA. Alternatively, glucose-imprinted polymers could be combined with glucose-binding proteins such as concanavilin A (ConA) to form supramolecular assemblies that are similarly responsive to glucose. Glucose-sensitive nanoparticle systems can also be engineered by combining $\mathrm{pH}$-sensitive polymers with the glucose-sensitive enzyme glucose oxidase, which enzymatically converts glucose to gluconic acid, producing a drop in $\mathrm{pH}$ in the nanoparticle microenvironment. $\mathbf{b}$ |The triggers can be integrated within a nanoparticle that is engineered to disassemble by either swelling or degrading in response to increased glucose levels, thus providing a mechanism by which the insulin cargo can be released and made bioavailable.

systems offer the potential to more accurately mimic the physiological response to changes in blood glucose levels and correspondingly modulate the kinetics of insulin release. Such improvements may provide tighter glycaemic control while minimizing the potential for hypoglycaemia. By combining advances in polymer engineering and nanotechnology, nanoparticle formulations can be engineered that can sense changes in their environment and disassemble to release their cargo $^{98}$ (FIG. 3). As noted above, the three most common glucose-sensing triggers are glucose oxidase, glucose-binding proteins and glucose-binding small molecules ${ }^{99}$ (FIG. 3a). Nanoparticle formulations using these glucose-responsive molecules can be engineered that detect environmental fluctuations in blood glucose levels and respond by releasing the insulin cargo through material degradation, disassembly or swelling ${ }^{100,101}$ (FIG. 3b).

As noted above, the specific enzymatic conversion of glucose to gluconic acid in biological environments (that is, in blood and urine $)^{55}$ by glucose oxidase is the most prevalent of the various glucose-sensing mechanisms described in the literature ${ }^{102}$. One early example utilizing this mechanism involved $\mathrm{pH}$-responsive insulin analogues that are insoluble at physiological $\mathrm{pH}$ but soluble under acidic conditions, allowing insulin release into the body ${ }^{103}$. When formulated with glucose oxidase and implanted in the body, hyperglycaemic conditions result in acidic microenvironments and insulin is therefore released to regulate blood glucose levels. More recently, material systems engineered to respond to acidic environments have been used as glucose-responsive platforms. For example, microgels composed of chitosan, glucose oxidase nanocapsules and insulin demonstrated glucose-dependent release of insulin and control of blood 


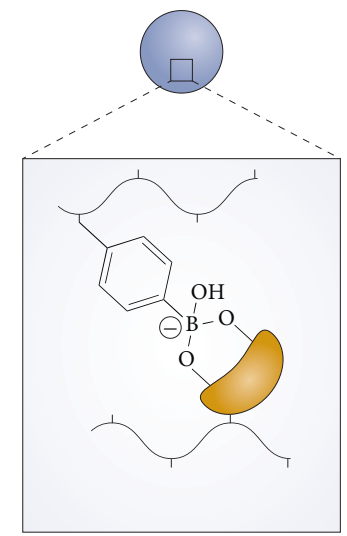

\section{$\uparrow$ [Glucose] Competitively binds to PBA}

Polymer breaks apart

\section{ConA-triggered release}

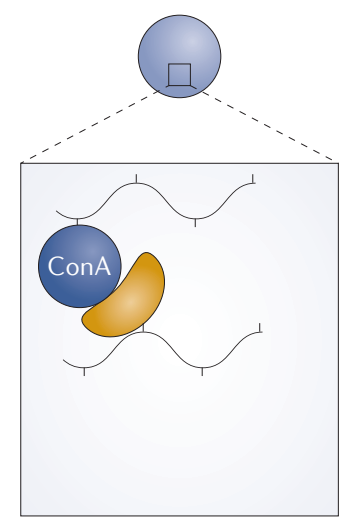

\section{$\uparrow$ [Glucose]} Competitively binds to ConA

Polymer breaks apart

Glucose oxidase-triggered release

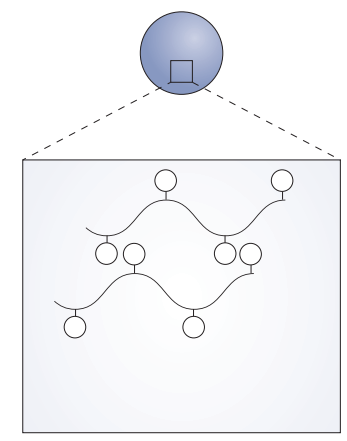

$\uparrow$ [Glucose], $\downarrow \mathrm{pH}$

$\overrightarrow{\text { Polymer breaks apart }}$

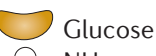

$\mathrm{NH}_{2}$
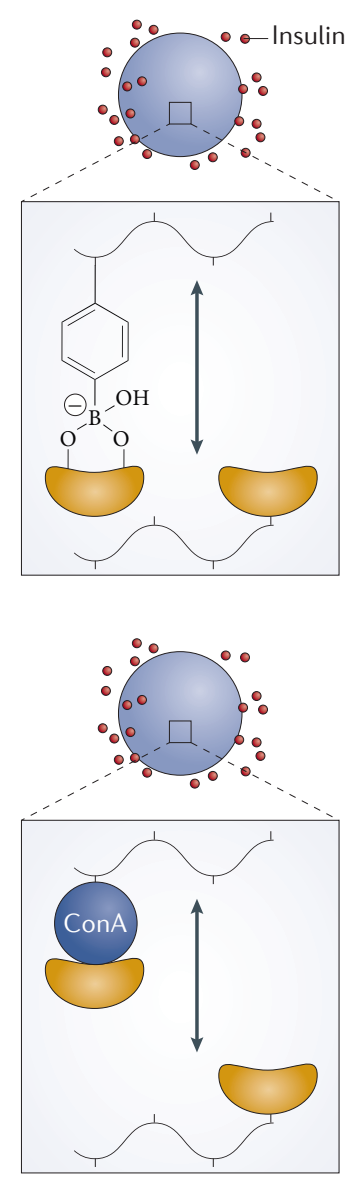

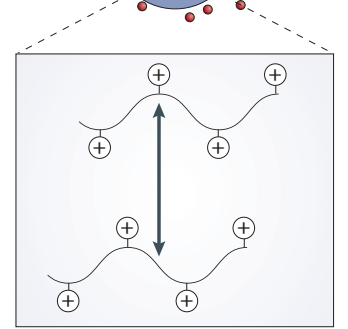

b

Glucose-mediated swelling
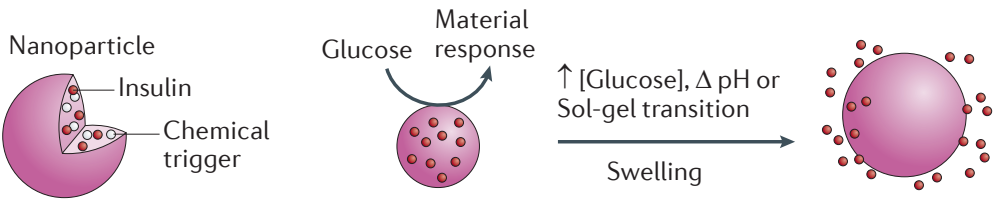

Glucose-mediated degradation
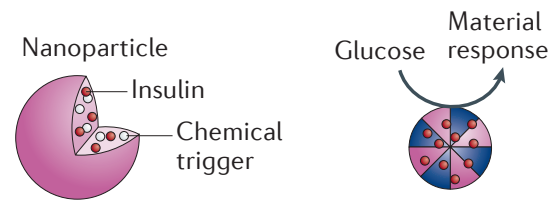

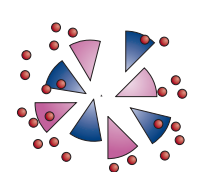

glucose in animal models ${ }^{104}$. Polymeric nanoparticles have been developed that erode under acidic conditions to release insulin cargos ${ }^{105,106}$. Based on this principle, a glucose-mediated insulin-delivering nanonetwork formulation was developed ${ }^{107}$. Many of these strategies have demonstrated pulsatile insulin release in vitro in response to changing glucose concentrations and euglycaemia in rodents from 10 to 295 days after the administration of a single dose. However, glucose oxidase-based systems can have slow and sometimes unpredictable response rates to changing glucose concentrations due to changes in temperature or oxygen concentration - that subsequently affect the translation of a change in glucose concentration into a shift in $\mathrm{pH}$.

Alternatively, efforts to develop non-enzymatic glucose-responsive materials and drug delivery systems have focused on the discovery of chemical moieties that specifically bind glucose. For example, glucosebinding moieties can be used as crosslinkers between polymers exhibiting glucose side chains, whereby free glucose competitively binds to the moiety to disrupt the crosslinks, leading to disassembly or swelling to release cargo $^{108}$ (FIG. 3b). One example involves the combination of glucose-modified insulin and the glucose-binding lectin, ConA, as an injectable conjugate that dissociates under increased glucose concentrations ${ }^{91,109}$. Although the responsive system remains to be demonstrated in vivo, modified insulins exhibit comparable activity to native insulin in rodents. Indeed, ConA formulations have garnered interest because they bind glucose with high specificity and affinity. However, a major challenge has been to overcome host immunological responses to non-native $\mathrm{ConA}^{110}$. Despite the immunological concerns, there is still great interest in developing such systems for clinical use. In fact, in 2010 Merck made an investment to develop a lectin-based glucose-responsive nanoparticle technology named 'Smart Insulin'111, which suggests there is growing industrial interest in glucoseresponsive formulations. Reports from the company suggest that Phase I trials may soon be underway to evaluate L-490 (Smart Insulin) in patients with diabetes ${ }^{112}$.

Small-molecule glucose binders such as PBA represent a chemical approach to glucose-responsive insulin delivery. For example, PBA has been formulated to function similarly to glucose-binding proteins as a gel with glucose-decorated polymers. Such a system can act as a sol-gel crosslinker ${ }^{113,114}$. An increase in glucose concentration leads to a decrease in the crosslinking density, resulting in swelling or erosion to its soluble form ${ }^{113}$. As glucose concentration decreases, the glucose exchange is reversed, and the borate-diol crosslinking is re-established. Two challenges to the use of PBA lie in its lack of specificity for glucose, as it has a higher affinity for other diols in the body such as fructose, and its $\mathrm{p} K_{\mathrm{a}}$ of $\sim 9$, which precludes efficient binding to glucose at physiological $\mathrm{pH}^{74}$. Efforts are underway to develop PBA derivatives that can function under physiological $\mathrm{pH}$ with improved glucose specificity ${ }^{115,116}$.

In recent years, several stimuli-responsive nanodevices have been described to be capable of responding to changes in $\mathrm{pH}^{117}$, shear pressure ${ }^{118}$ and external stimuli 
(that is, light ${ }^{119}$, magnetic forces ${ }^{120}$ or ultrasonic waves ${ }^{121}$ ) to release drugs. For example, a nanonetwork of insulin with PLGA (poly(lactic-co-glycolic acid)) nanoparticles releases insulin at basal levels and releases a burst of insulin upon exposure to ultrasound ${ }^{121}$. As the field of nanotechnology matures, new opportunities may emerge for the development of insulin-delivering nanodevices that better mimic the physiological needs of the patient.

In summary, the most evaluated approaches for developing glucose-responsive insulin delivery have been those based on glucose oxidase owing to its high specificity for glucose, its current usage in glucose sensors and the wide array of $\mathrm{pH}$-responsive materials. However, the enzymatic conversion of glucose remains unreliable and slow, and the release of insulin from these nanoparticles is indirectly related to glucose concentration. Glucose-binding proteins provide high specificity and binding to glucose; however, limited progress has been made towards eliminating the foreign body response. Small-molecule binders currently lack specificity for glucose but new approaches such as multiplexing PBAs are being investigated to address this concern.

Non-invasive delivery. The development of non-invasive methods for insulin administration has the potential to improve patient compliance and reduce complications associated with poor glycaemic control. Oral, inhalable and transdermal delivery can provide painless and simple methods relative to traditional insulin injections ${ }^{18}$. However, poor and unpredictable bioavailability has limited the success of insulin delivery via these alternative routes. This is due in part to the harsh environment of the gastrointestinal tract, variable and unpredictable inhalation efficiency, and limited transport across epithelial barriers ${ }^{93}$. Nanotechnology has been used to address these challenges and develop non-traditional delivery routes ${ }^{18}$ (FIG. 4).

The oral route is patient-friendly, as the ingestion of solid tablets or liquids is non-invasive and relatively discrete $^{93}$. However, orally delivered insulin must survive the harsh enzymatic environment of the gut and be transported across the intestinal epithelial barrier before it can enter the bloodstream to have a glucose-lowering effect ${ }^{93}$. Nanoparticles have been used as protective carriers against enzymatic and hydrolytic degradation for various drugs, including insulin ${ }^{122}$. Transport across epithelial barriers can occur via passive (that is, transcellular or paracellular) diffusion ${ }^{97,123}$, active transport (that is, receptor-mediated transport or involving membrane-derived vesicles and membrane-bound carriers) ${ }^{124}$, endocytosis (that is, adsorptive-mediated endocytosis and fluid-phase endocytosis $)^{125}$ and Microfold cell ( $\mathrm{M}$ cell) antigen sampling ${ }^{126}$. Formulations have been developed to stimulate paracellular transport ${ }^{127}$, and nanoparticles can be decorated with ligands to facilitate receptor-mediated transcytosis across epithelial barri$\mathrm{ers}^{128}$. Recently, insulin-loaded polymeric PLGA nanoparticles functionalized with Fc fragments on the surface were reported to target the neonatal Fc receptor in the intestinal epithelium ${ }^{128}$. When tested in vivo in mice, these Fc-fragment-decorated nanoparticles improved transport across the intestinal epithelium, resulting in a tenfold higher mean absorption efficiency compared to non-targeted nanoparticles ${ }^{128}$. Importantly, a biologically relevant insulin dose of $1.1 \mathrm{U}$ per $\mathrm{kg}$ was able to reduce blood glucose levels in a healthy mouse, which indicates that the delivered insulin was biologically active and functional ${ }^{128}$. Although these findings are promising, the utility of oral insulin nanoparticles may be limited to replacing injections of long-acting insulin, whereas replacing fast-acting insulin will require more predictable insulin absorption profiles.

The airways, which have a large surface area, can also be used to deliver drugs, and offer the advantages of a relatively mild environment (neutral $\mathrm{pH}$ and low enzyme concentrations) compared to the gastrointestinal tract, as well as access to the underlying vascular and lymphatic systems ${ }^{129,130}$. Nanoparticle formulations have the potential to enhance inhaled drug delivery as a result of improved distribution in the airways, regulation of drug release rates, their ability to penetrate the mucosal barrier ${ }^{131}$ and transport across the epithelial barrier ${ }^{132}$. Inhaled insulin nanoparticles have demonstrated sustained insulin activity (18 hours) over standard subcutaneous injections ( 2 hours) in diabetic rats ${ }^{133}$. Exubera, a dry powder inhaled insulin formulation developed by Pfizer and approved in 2006, provided the first non-injectable insulin option for patients with diabetes. Inhaled insulin offered similar bioactivity to subcutaneously injected fast-acting insulin ${ }^{134}$, but was ultimately withdrawn from the market owing to poor sales, which have been attributed to added costs, a bulky device and an FDA-imposed requirement that patients must undergo regular lung function tests to ensure they are receiving proper insulin bioavailability ${ }^{14,135,136}$. Recently, a more patient-friendly formulation, Afrezza (MannKind Corporation), received marketing approval from the FDA to improve glycaemic control in adults with type 2 diabetes ${ }^{137}$. Afrezza is an ultra-fastacting inhalable insulin prepared as microspheres that is administered before meals. In clinical studies, it has been shown to lower glycated haemoglobin (HbA1c) levels in patients with type 1 and type 2 diabetes compared to standard treatment protocols ${ }^{138}$.

Alternatively, the transdermal route can be used to actively or passively transport nanomaterials through the skin for diabetes therapy. Transdermal drug delivery has the potential to facilitate sustained delivery of substantial payloads $^{94}$. However, molecules that are large or hydrophilic, such as insulin, suffer from poor absorption owing to the very low permeability of the stratum corneum ${ }^{139}$. One approach has been to explore gold nanorods ( $27 \times 66 \mathrm{~nm}$ in size) as vehicles to deliver insulin transdermally through a paracellular pathway ${ }^{140}$. In addition, permeation enhancers have enabled transdermal delivery of other nanoparticles ${ }^{141}$. Other approaches utilize a more active method, such as ultrasonication or heat to locally increase the permeability of the skin in order to improve delivery ${ }^{142}$. In diabetic rodents, these systems have demonstrated blood glucose suppression for up to 10 hours $^{140}$ and peak insulin concentrations for around 4 hours $^{143}$. 


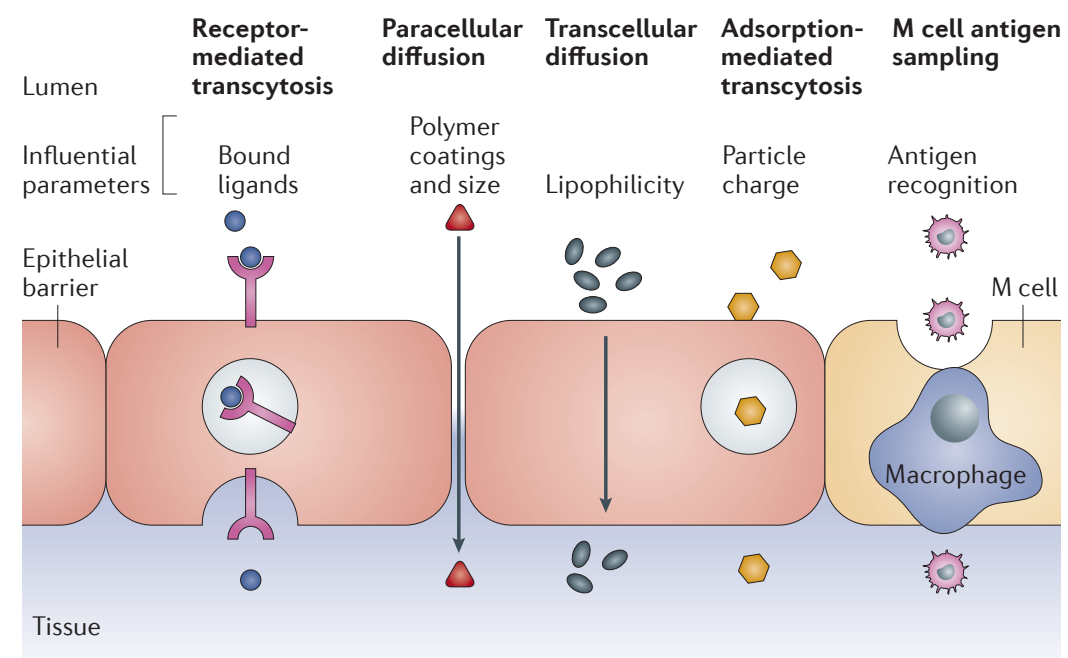

Figure 4 | Development of patient-friendly insulin delivery nanoparticle formulations. Various properties of nanoparticles can be tailored to exploit transepithelial transport mechanisms to facilitate systemic insulin delivery. Nanoparticles prepared with bound ligands for specialized receptors expressed on epithelial cell surfaces can be transcytosed across epithelial barriers. Ultrasmall nanoparticles with hydrophilic coatings can exploit paracellular diffusion to bypass epithelial barriers. Nanoparticles with tuned lipophilic physicochemical properties can permeate across epithelial barriers through a transcellular pathway. Cationic charged nanoparticles can exploit the adsorption-mediated transcytosis pathway for transport across epithelial barriers. Finally, nanoparticles that are transported across epithelial barriers by antigen sampling Microfold cells (M cells) could be developed.

With the advent of novel routes of insulin delivery using nanoparticles, the safety and loading capacity of formulations must be considered. Insulin loading is typically poor in water-insoluble nanoparticles, with insulin representing a small percentage of the total formulation by weight ${ }^{128}$. As such, a substantial amount of material will need to be administered to achieve sufficient insulin delivery, and the formulation material will need to be suitably cleared following repeated dosing to allow for long-term treatment.

\section{Non-insulin-based therapies}

Cell-based therapy for diabetes involves the regeneration of $\beta$-cells, reprogramming of native cells to secrete insulin or the transplantation of insulin-producing cells to restore insulin production in response to glucose level changes ${ }^{144}$. The introduction of new insulin-producing cells in the body can lead to a foreign body response and transplant rejection or generate an innate immune response against these cells; long-term protection against these responses is necessary to ensure the survival and function of transplanted insulin-producing cells ${ }^{144}$. Nanoparticles hold the potential to address some of these challenges.

Exogenous cell therapy, typically in the form of islet transplantation, was introduced as an approach to restore normoglycaemia in patients with type 1 diabetes in the early $1970 \mathrm{~s}^{145}$. However, owing to host rejection of transplanted cells, limited amounts of donor cells and the extensive immunosuppressive therapy needed to address it, the clinical application of islet transplantation has been limited ${ }^{144}$. Over the past four decades, efforts have been made to develop an improved bioartificial pancreas that alleviates the need for immunosuppressive therapies ${ }^{146}$. Nanotechnology is now being developed for more advanced engineering of complex tissues ${ }^{22}$. For example, nanotechnology has been used to isolate and protect transplanted cells from the immune system while allowing sufficient diffusion of oxygen, glucose, insulin and other necessary nutrients ${ }^{147}$. Towards this goal, various conformal coating approaches, including layer-by-layer polymer deposition ${ }^{148}$, polyion complex formation ${ }^{149}$ and chemical reactions of polymers ${ }^{150}$, have been applied to islets to produce nano-thin coatings that may protect islet activity without inhibiting their function ${ }^{148}$. One barrier in translating these technologies to the clinic remains the lack of encapsulating materials that can avoid host recognition and subsequent foreign body responses ${ }^{151}$. Future advancement of islet encapsulation requires that materials and devices be developed such that encapsulated cells can maintain function and viability while avoiding fibrosis ${ }^{152}$.

Gene therapy to either express or silence specific genes involved in the immune response is an alternative strategy that circumvents the immune response to cell therapies ${ }^{153}$. Nanoparticles have been developed to protect and deliver nucleic acids to target cells ${ }^{154}$. For example, polymeric nanoparticles have been used to deliver DNA encoding interleukin-10 (IL-10) and IL-4 to white blood cells to suppress the T cell response against remaining innate islet cells in animal models of early diabetes, preventing the development of diabetes in $75 \%$ of animals ${ }^{155}$. Alternatively, the gene encoding glucagon-like peptide 1 has been delivered via nanoparticles to boost insulin secretion and islet viability ${ }^{156}$.

One interesting example describes bioengineered cells containing SPIONs that have been demonstrated to produce insulin upon induction of hyperthermia through the external application of alternating magnetic currents. In this study, it was demonstrated that the cells were able to generate insulin on demand in vivo to regulate blood glucose levels ${ }^{120}$. Although the complexity of this specific system may delay its clinical translation, the concept of using non-invasive external triggers to regulate insulin production and release is exciting and opens new opportunities for engineering an automated synthetic pancreas device.

Finally, vaccines have been investigated as a longterm strategy to prevent the autoimmune destruction of $\beta$-cells in patients with type 1 diabetes. Nanotechnology in general has been used to improve the development of vaccines for a number of diseases ${ }^{157}$. It has been shown to alleviate the need for antigen adjuvants ${ }^{158}$ and can direct antigens to specific sites of the body ${ }^{159}$. Nanotechnology can also improve the potency of antigens ${ }^{160}$, provide a physical platform for the use of combinations of antigens ${ }^{161}$ and enable the delivery of self-replicating and RNA-based antigens ${ }^{162,163}$. Specifically, diabetes vaccine development efforts have focused on blunting the cytotoxic $\mathrm{T}$ cell immune response against $\beta$-cells without compromising global immunity ${ }^{5}$. Using a non-obese diabetic mouse model, magnetic nanoparticles coated 


\section{Closed-loop}

A form of insulin replacement therapy whereby the required insulin is automatically determined and the proper insulin dosage is delivered with minimal patient involvement. with appropriate peptide-major histocompatibility complexes (pMHC-NPs) were shown to expand a population of naive low-avidity autoreactive $\mathrm{CD}^{+} \mathrm{T}$ cells into memory-like autoregulatory cells, to prevent and reverse type 1 diabetes in $75 \%$ of mice ${ }^{164}$.

\section{Conclusions and outlook}

The development of nanotechnology for the management of diabetes has only recently begun, but is occurring at a rapid pace owing to inspiration and adaptation from successes in treatments for other diseases. These include cancer ${ }^{165-167}$, for which the first nanoparticlebased therapy, a pegylated liposome nanoparticle formulation loaded with the chemotherapeutic agent doxorubicin, received FDA approval in 1995 (REF. 168). Since then, the pipeline of nanomedicines for cancer indications has expanded considerably, with more than 20 different formulations currently under clinical investigation $^{169}$. Nanotechnology has also been developed for the management of cardiovascular disease ${ }^{170,171}$; for example, nanoparticles have been used to deliver MRI contrast agents for the monitoring of acute myocardial infraction in human patients ${ }^{172}$. As a result, there is already an extensive toolbox of promising and clinically applicable nanotechnology-based formulations ${ }^{173}$.

Non-invasive monitoring of disease progression and blood glucose levels, glucose-responsive and patientfriendly insulin, and improved immune modulation for cell-based therapies are among the advances developed to date. However, the bar for success is high, as diabetes management is well established with advanced control algorithms using continuous glucose monitors and insulin pumps in outpatient clinical studies ${ }^{13}$. The long-term safety of nanotechnology is also under increased scrutiny ${ }^{173}$. Indeed, the FDA recently issued guidance to help foster the safe development of nanotechnology-based products for clinical use ${ }^{174}$. Safety and long-term performance must be fully evaluated in the design of diabetes therapeutics and diagnostics, especially for materials that are not degraded or cleared from the body.

We envision promising opportunities in the development of closed-loop glucose sensing and insulin-delivering nanoparticle formulations. Further development of new glucose-sensing molecules that can serve as triggering mechanisms for both sensors and glucose-responsive materials will be key to the advancement of this technology. The next generation of nanosensors and integrated glucose-mediated insulin delivery formulations will need to demonstrate increased sensitivity and specificity to glucose. Specifically, a major challenge remains with prolonged lag times for response to increased blood glucose levels. A potential solution may rest in the development of new glucose-responsive moieties with stronger association constants, as well as materials containing these binding domains. The next generation of glucose sensor technologies will need to be more consistent and reliable, with less drift resulting from sensor degradation or failure. A tight coupling between glucose sensing and insulin delivery is needed to effectively control blood glucose. The implementation of glucose-mediated insulin-delivering technologies into the clinical setting will probably hinge on innovations that help to reduce the lag between sensing and therapeutic delivery, as well as increasing the response rate to changes in glucose levels.

Cell-based therapy is another area in which nanotechnology may have an important role in reducing the immune response to the new insulin-producing cells. Nanoparticles show considerable promise as agents for delivering nucleic acid therapeutics ${ }^{175}$, and advances are likely to be made with the development of improved cellular targeting strategies. These may be useful in therapies that involve the reprogramming of endogenous cells into islet-like cells, as well as in the development of transplanted islets ${ }^{176}$.

In summary, we expect nanotechnology to play an important part in improving the management of diabetes within the next decade. The emergence of FDAapproved nanotechnology formulations coupled with the clinical success of insulin-delivering technologies through the pulmonary route is encouraging. In our view, the greatest need and also the highest clinical potential for nanotechnology-based diabetes therapy lies in the development of robust glucose-sensitive nanoparticles and nanodevices for integration into sensors, and the development of integrated glucose-sensing and insulin-delivering nanoformulations.
1. American Diabetes Association. Standards of medical care in diabetes - 2014. Diabetes Care 37, S14-S80 (2014).

2. Shaw, J. E., Sicree, R. A. \& Zimmet, P. Z. Global estimates of the prevalence of diabetes for 2010 and 2030. Diabetes Res. Clin. Pract. 87, 4-14 (2010).

3. Zhang, P. et al. Global healthcare expenditure on diabetes for 2010 and 2030. Diabetes Res. Clin. Pract. 87, 293-301 (2010).

4. Dabelea, D. The accelerating epidemic of childhood diabetes. Lancet 373, 1999-2000 (2009).

5. Lieberman, S. M. \& DiLorenzo, T. P. A comprehensive guide to antibody and T-cell responses in type 1 diabetes. Tissue Antigens 62, 359-377 (2003).

6. Ismail-Beigi, F. Glycemic management of type 2 diabetes mellitus. N. Engl. J. Med. 366, 1319-1327 (2012).

7. Donath, M. Y. \& Shoelson, S. E. Type 2 diabetes as an inflammatory disease. Nature Rev. Immunol. 11, 98-107 (2011).
8. Pickup, J. C. Management of diabetes mellitus: is the pump mightier than the pen? Nature Rev. Endocrinol. 8, 425-433 (2012)

Excellent review comparing and contrasting the clinical benefits for the use of insulin pumps versus injectable insulin formulations.

9. American Diabetes Association. Standards of medical care in diabetes-2013. Diabetes Care 36 (Suppl. 1), 11-66 (2013)

10. Sarwar, N. et al. Diabetes mellitus, fasting blood glucose concentration, and risk of vascular disease: a collaborative meta-analysis of 102 prospective studies. Lancet 375, 2215-2222 (2010).

11. Schulman, R. C. et al. Association of glycemic control parameters with clinical outcomes in chronic critical illness. Endocr. Pract. 20, 884-893 (2014).

12. Berenson, D. F., Weiss, A. R., Wan, Z. L. \& Weiss, M. A Insulin analogs for the treatment of diabetes mellitus: therapeutic applications of protein engineering Ann. NY Acad. Sci. 1243, E40-E54 (2011).

13. Tamborlane, W. V. et al. Continuous glucose monitoring and intensive treatment of type 1 diabetes. N. Engl. J. Med. 359, 1464-1476 (2008).
14. Heinemann, L. New ways of insulin delivery. Int. J. Clin. Pract. 65 (Suppl. 170), 31-46 (2011)

Excellent review summarizing the advances towards developing insulin analogues with tailored pharmacokinetics.

15. Pickup, J. C. Insulin-pump therapy for type diabetes mellitus. N. Engl. J. Med. 366 1616-1624 (2012).

16. Russell, S. J. et al. Outpatient glycemic control with a bionic pancreas in type 1 diabetes. N. Engl. J. Med. 371, 313-325 (2014).

A Phase II clinical trial that demonstrates the improved efficacy and safety of a wearable, automated, bihormonal, bionic pancreas dual hormone insulin pump compared to a conventional insulin pump.

17. Resnick, H. E., Foster, G. L., Bardsley, J. \& Ratner, R. E. Achievement of American Diabetes Association clinical practice recommendations among US adults with diabetes, 1999-2002: the National Health and Nutrition Examination Survey. Diabetes Care 29, 531-537 (2006). 
18. Owens, D. R. New horizons - alternative routes for insulin therapy. Nature Rev. Drug Discov. 1, 529-540 (2002).

19. Mehanna, A. Antidiabetic agents: past, present and future. Future Med. Chem. 5, 411-430 (2013).

20. Weissleder, R. \& Pittet, M. J. Imaging in the era of molecular oncology. Nature 452, 580-589 (2008).

21. Whitesides, G. M. The 'right' size in nanobiotechnology. Nature Biotech. 21, 1161-1165 (2003).

22. Dvir, T., Timko, B. P., Kohane, D. S. \& Langer, R. Nanotechnological strategies for engineering complex tissues. Nature Nanotech. 6, 13-22 (2011).

23. Schroeder, A. et al. Treating metastatic cancer with nanotechnology. Nature Rev. Cancer 12, 39-50 (2012).

24. LaVan, D. A., Lynn, D. M. \& Langer, R. Moving smaller in drug discovery and delivery. Nature Rev. Drug Discov. 1, 77-84 (2002).

25. McNeil, S. E. Unique benefits of nanotechnology to drug delivery and diagnostics. Methods Mol. Biol. 697, 3-8 (2011)

26. Venkatraman, S. S., Ma, L. L., Natarajan, J. V. \& Chattopadhyay, S. Polymer- and liposome-based nanoparticles in targeted drug delivery. Front. Biosci. 2, 801-814 (2010)

27. Stinchcombe, T. E. Nanoparticle albumin-bound paclitaxel: a novel Cremphor-EL-free formulation of paclitaxel. Nanomed. 2, 415-423 (2007).

28. Barbas, A. S., Mi, J., Clary, B. M. \& White, R. R. Aptamer applications for targeted cancer therapy. Future Oncol. 6, 1117-1126 (2010).

29. Chiu, G. N. et al. Lipid-based nanoparticulate systems for the delivery of anti-cancer drug cocktails: Implications on pharmacokinetics and drug toxicities. Curr. Drug Metab. 10, 861-874 (2009)

30. Schroeder, A., Levins, C. G., Cortez, C., Langer, R. \& Anderson, D. G. Lipid-based nanotherapeutics for siRNA delivery. J. Intern. Med. 267, 9-21 (2010)

31. Veiseh, O., Gunn, J. W. \& Zhang, M. Design and fabrication of magnetic nanoparticles for targeted drug delivery and imaging. Adv. Drug Deliv. Rev. 62, 284-304 (2010)

32. Boisselier, E. \& Astruc, D. Gold nanoparticles in nanomedicine: preparations, imaging diagnostics, therapies and toxicity. Chem. Soc. Rev. 38, 1759-1782 (2009).

33. Leonard, F. \& Talin, A. A. Electrical contacts to one- and two-dimensional nanomaterials. Nature Nanotech. 6, 773-783 (2011).

34. Pickup, J. C., Zhi, Z. L., Khan, F., Saxl, T. \& Birch, D. J. Nanomedicine and its potential in diabetes research and practice. Diabetes Metab. Res. Rev. 24, 604-610 (2008).

35. Naesens, M. \& Sarwal, M. M. Molecular diagnostics in transplantation. Nature Rev. Nephrol. 6, 614-628 (2010).

Excellent review highlighting the use of molecula imaging tools to monitor cell transplantation outcomes.

36. Matveyenko, A. V. \& Butler, P. C. Relationship between $\beta$-cell mass and diabetes onset. Diabetes Obes. Metab. 10 (Suppl. 4), 23-31 (2008).

37. Reiner, T. et al. Accurate measurement of pancreatic islet $\beta$-cell mass using a second-generation fluorescent exendin-4 analog. Proc. Natl Acad. Sci. USA 108, 12815-12820 (2011).

38. Moore, A., Bonner-Weir, S. \& Weissleder, R. Noninvasive in vivo measurement of $\beta$-cell mass in mouse model of diabetes. Diabetes 50, 2231-2236 (2001).

39. Malaisse, W. J. \& Maedler, K. Imaging of the $\beta$-cells of the islets of Langerhans. Diabetes Res. Clin. Pract. 98, 11-18 (2012).

40. Lamprianou, S. et al. High-resolution magnetic resonance imaging quantitatively detects individual pancreatic islets. Diabetes 60, 2853-2860 (2011).

41. Leibiger, I. B., Caicedo, A. \& Berggren, P. O. Non-invasive in vivo imaging of pancreatic $\beta$-cell function and survival - a perspective. Acta Physiol. 204, 178-185 (2012).

42. Fu, W., Wojtkiewicz, G., Weissleder, R., Benoist, C. \& Mathis, D. Early window of diabetes determinism in NOD mice, dependent on the complement receptor $\mathrm{CRIg}$, identified by noninvasive imaging. Nature Immunol. 13, 361-368 (2012).

43. Li, D. et al. Imaging dynamic insulin release using a fluorescent zinc indicator for monitoring induced exocytotic release (ZIMIR). Proc. Natl Acad. Sci. USA 108, 21063-21068 (2011).
44. Lee, N. et al. Magnetosome-like ferrimagnetic iron oxide nanocubes for highly sensitive MRI of single cells and transplanted pancreatic islets. Proc. Natl Acad. Sci. USA 108, 2662-2667 (2011).

45. Medarova, Z., Evgenov, N. V., Dai, G., Bonner-Weir, S. $\&$ Moore, A. In vivo multimodal imaging of transplanted pancreatic islets. Nature Protoc. 1 , 429-435 (2006).

46. Medarova, Z. \& Moore, A. MRI as a tool to monitor islet transplantation. Nature Rev. Endocrinol. 5 444-452 (2009).

47. Andralojc, K. et al. Obstacles on the way to the clinical visualisation of $\beta$ cells: looking for the Aeneas of molecular imaging to navigate between Scylla and Charybdis. Diabetologia 55, 1247-1257 (2012)

48. Wu, Z. \& Kandeel, F. Radionuclide probes for molecular imaging of pancreatic $\beta$-cells. Adv. Drug Delivery Rev. 62, 1125-1138 (2010).

49. Sun, C., Lee, J. S. \& Zhang, M. Magnetic nanoparticles in MR imaging and drug delivery. Adv. Drug Delivery Rev. 60, 1252-1265 (2008).

50. Gaglia, J. L. et al. Noninvasive imaging of pancreatic islet inflammation in type $1 \mathrm{~A}$ diabetes patients. J. Clin. Invest. 121, 442-445 (2011). Clinical study that demonstrates feasibility of using ferumoxtran-10 - a dextran-coated iron oxide nanoparticle to monitor pancreatitis.

51. Mauras, N., Fox, L., Englert, K. \& Beck, R. W. Continuous glucose monitoring in type 1 diabetes. Endocr. 43, 41-50 (2013).

52. Bernhardt, P. Self-monitoring blood glucose tes systems for over-the-counter use. U.S. Food and Drug Administration [online]

53. Scognamiglio, V. Nanotechnology in glucose monitoring: advances and challenges in the last 10 years. Biosens. Bioelectron. 47C, 12-25 (2013)

54. Ansari, S. G. et al. Glucose sensor based on nanobaskets of tin oxide templated in porous alumina by plasma enhanced CVD. Biosens. Bioelectron. 23 1838-1842 (2008)

55. Bankar, S. B., Bule, M. V., Singhal, R. S. \& Ananthanarayan, L. Glucose oxidase - an overview. Biotechnol. Adv. 27, 489-501 (2009).

56. Ricci, F. et al. Novel planar glucose biosensors for continuous monitoring use. Biosens. Bioelectron 20, 1993-2000 (2005).

57. Zhang, S., Wang, N., Yu, H., Niu, Y. \& Sun, C. Covalent attachment of glucose oxidase to an $\mathrm{Au}$ electrode modified with gold nanoparticles for use as glucose biosensor. Bioelectrochemistry 67, 15-22 (2005)

58. Tang, $\mathrm{H}$. et al. Amperometric glucose biosensor based on adsorption of glucose oxidase at platinum nanoparticle-modified carbon nanotube electrode. Anal. Biochem. 331, 89-97 (2004)

59. Claussen, J. C. et al. Electrochemical glucose biosensor of platinum nanospheres connected by carbon nanotubes. J. Diabetes Sci. Technol 4 312-319 (2010).

60. Hoedemaekers, C. W., Klein Gunnewiek, J. M. Prinsen, M. A., Willems, J. L. \& Van der Hoeven, J. G. Accuracy of bedside glucose measurement from three glucometers in critically ill patients. Crit. Care Med. 36, 3062-3066 (2008)

61. Hussain, A. M., Sarangi, S. N., Kesarwani, J. A. \& Sahu, S. N. Au-nanocluster emission based glucose sensing. Biosens. Bioelectron. 29, 60-65 (2011).

62. Jiang, L. C. \& Zhang, W. D. A highly sensitive nonenzymatic glucose sensor based on $\mathrm{CuO}$ nanoparticles-modified carbon nanotube electrode. Biosens. Bioelectron. 25, 1402-1407 (2010).

63. Yang, Y. et al. Glucose sensors based on electrodeposition of molecularly imprinted polymeric micelles: a novel strategy for MIP sensors. Biosens. Bioelectron. 26, 2607-2612 (2011)

64. Yang, J., Jiang, L. C., Zhang, W. D. \& Gunasekaran, S. A highly sensitive non-enzymatic glucose sensor based on a simple two-step electrodeposition of cupric oxide $(\mathrm{CuO})$ nanoparticles onto multi-walled carbon nanotube arrays. Talanta $\mathbf{8 2}, 25-33$ (2010)

65. Pickup, J. C., Hussain, F., Evans, N. D., Rolinski, O. J. \& Birch, D. J. Fluorescence-based glucose sensors. Biosens. Bioelectron. 20, 2555-2565 (2005).

66. Nielsen, J. K. et al. Clinical evaluation of a transcutaneous interrogated fluorescence lifetime-based microsensor for continuous glucose reading. J. Diabetes Sci. Technol. 3, 98-109 (2009).
67. Schultz, J S. Mansouri, S. \& Coldstein, I. J. Affinity sensor: a new technique for developing implantable sensors for glucose and other metabolites. Diabetes Care 5, 245-253 (1982).

68. Liao, K. C et al. Percutaneous fiber-optic sensor for chronic glucose monitoring in vivo. Biosens. Bioelectron. 23, 1458-1465 (2008)

69. Chen, P. C., Wan, L. S., Ke, B. B. \& Xu, Z. K. Honeycomb-patterned film segregated with phenylboronic acid for glucose sensing. Langmuir 27, 12597-12605 (2011).

70. Kataoka, K., Hisamitsu, I., Sayama, N., Okano, T. \& Sakurai, Y. Novel sensing system for glucose based on the complex formation between phenylborate and fluorescent diol compounds. J. Biochem. 117 1145-1147 (1995).

71. Parmpi, P. \& Kofinas, P. Biomimetic glucose recognition using molecularly imprinted polymer hydrogels. Biomaterials 25, 1969-1973 (2004)

72. Edelman, G. M. et al. The covalent and threedimensional structure of concanavalin A. Proc. Natl Acad. Sci. USA 69, 2580-2584 (1972).

73. Ge, X. et al. Comparing the performance of the optical glucose assay based on glucose binding protein with high-performance anion-exchange chromatography with pulsed electrochemical detection: efforts to design a low-cost point-of-care glucose sensor. J. Diabetes Sci. Technol. 1, 864-872 (2007)

74. Bull, S. D. et al. Exploiting the reversible covalent bonding of boronic acids: recognition, sensing and assembly. Accounts Chem. Res. 46, 312-326 (2013).

75. Barone, P. W. \& Strano, M. S. Single walled carbon nanotubes as reporters for the optical detection of glucose. J. Diabetes Sci. Technol. 3, 242-252 (2009).

76. Yum, K., McNicholas, T. P., Mu, B. \& Strano, M. S Single-walled carbon nanotube-based near-infrared optical glucose sensors toward in vivo continuous glucose monitoring. J. Diabetes Sci. Technol. 7 72-87 (2013)

77. Balaconis, M. K., Billingsley, K., Dubach, M. J., Cash, K. J. \& Clark, H. A. The design and development of fluorescent nano-optodes for in vivo glucose monitoring. J. Diabetes Sci. Technol. 5, 68-75 (2011).

78. Billingsley, K. et al. Fluorescent nano-optodes for glucose detection. Anal. Chem. 82, 3707-3713 (2010).

79. Klonoff, D. C. Overview of fluorescence glucose sensing: a technology with a bright future. J. Diabetes Sci. Technol. 6, 1242-1250 (2012).

80. Barone, P. W., Baik, S., Heller, D. A. \& Strano, M. S. Near-infrared optical sensors based on single-walled carbon nanotubes. Nature Mater. 4, 86-92 (2005).

81. Barone, P. W., Parker, R. S. \& Strano, M. S. In vivo fluorescence detection of glucose using a single-walled carbon nanotube optical sensor: design, fluorophore properties, advantages, and disadvantages. Anal. Chem. 77, 7556-7562 (2005).

82. Iverson, N. M. et al. In vivo biosensing via tissuelocalizable near-infrared-fluorescent single-walled carbon nanotubes. Nature Nanotech. 8, 873-880 (2013).

83. Saito, N. et al. Safe clinical use of carbon nanotubes as innovative biomaterials. Chem. Rev. 114, 6040-6079 (2014).

84. Schipper, M. L. et al. A pilot toxicology study of single-walled carbon nanotubes in a small sample of mice. Nature Nanotech. 3, 216-221 (2008)

85. Liu, Z. et al. Circulation and long-term fate of functionalized, biocompatible single-walled carbon nanotubes in mice probed by Raman spectroscopy. Proc. Natl Acad. Sci. USA 105, 1410-1415 (2008)

86. Khalil, O. S. in Glucose Sensing Vol. 11 (eds Geddes, C. D. \& Lakowicz, J. R.) (Springer Science, 2006)

87. Vashist, S. K. Non-invasive glucose monitoring technology in diabetes management: a review. Anal. Chim. Acta 750, 16-27 (2012).

88. Krol, S., Ellis-Behnke, R. \& Marchetti, P. Nanomedicine for treatment of diabetes in an aging population: state-of-the-art and future developments. Nanomed. 8 (Suppl. 1), 69-76 (2012).

89. Zhi, Z. L., Khan, F. \& Pickup, J. C. Multilayer nanoencapsulation: a nanomedicine technology for diabetes research and management. Diabetes Res. Clin. Pract. 100, 162-169 (2013). 
90. Peng O Zhang Z R Gong T Chen, G. O. \& Sun, X A rapid-acting, long-acting insulin formulation based on a phospholipid complex loaded PHBHHx nanoparticles. Biomaterials 33, 1583-1588 (2012).

91. Brownlee, M. \& Cerami, A. A glucose-controlled insulin-delivery system: semisynthetic insulin bound to lectin. Science 206, 1190-1191 (1979). First example of a glucose-responsive insulin formulation

92. Zhao, Y Trewyn, B. G. Slowing I I \& Lin V S. Mesoporous silica nanoparticle-based double drug delivery system for glucose-responsive controlled release of insulin and cyclic AMP. J. Am. Chem. Soc 131, 8398-8400 (2009)

93. Ensign, L. M., Cone, R. \& Hanes, J. Oral drug delivery with polymeric nanoparticles: the gastrointestinal mucus barriers. Adv. Drug Deliv. Rev. 64, 557-570 (2012).

94. Pegoraro, C., Macneil, S. \& Battaglia, G. Transdermal drug delivery: from micro to nano. Nanoscale 4 , 1881-1894 (2012).

95. McMahon, G. T. \& Arky, R. A. Inhaled insulin for diabetes mellitus. M. Engl. J. Med. 356, 497-502 (2007).

96. Weidemaier K et al Multi-day pre-clinical demonstration of glucose/galactose binding proteinbased fiber optic sensor. Biosens. Bioelectron. 26, 4117-4123 (2011)

97. Sheridan, C. Proof of concept for next-generation nanoparticle drugs in humans. Nature Biotech. 30 471-473 (2012)

98. Fleige, E., Quadir, M. A. \& Haag, R. Stimuli-responsive polymeric nanocarriers for the controlled transport of active compounds: concepts and applications. Adv. Drug Delivery Rev. 64, 866-884 (2012).

99. Qiu, Y. \& Park, K. Environment-sensitive hydrogels for drug delivery. Adv. Drug Deliv. Rev. 53, 321-339 (2001).

100. Stuart, M. A. et al. Emerging applications of stimuliresponsive polymer materials. Nature Mater. $\mathbf{9}$, 101-113 (2010)

101. Wu, W., Mitra, N., Yan, E. C. \& Zhou, S. Multifunctional hybrid nanogel for integration of optical glucose sensing and self-regulated insulin release at physiological pH. ACS Nano 4, 4831-4839 (2010).

102. Ferri, S., Kojima, K. \& Sode, K. Review of glucose oxidases and glucose dehydrogenases: a bird's eye view of glucose sensing enzymes. J. Diabetes Sci. Technol. 5, 1068-1076 (2011).

103. Fischel-Ghodsian, F., Brown, L., Mathiowitz, E. Brandenburg, D. \& Langer, R. Enzymatically controlled drug delivery. Proc. Natl Acad. Sci. USA 85, 2403-2406 (1988).

104. Gu, Z. et al. Glucose-responsive microgels integrated with enzyme nanocapsules for closed-loop insulin delivery. ACS Nano 7, 6758-6766 (2013).

105. Luo, J. et al. Super long-term glycemic control in diabetic rats by glucose-sensitive LbL films constructed of supramolecular insulin assembly. Biomaterials 33, 8733-8742 (2012).

106. Qi, W. et al. Triggered release of insulin from glucosesensitive enzyme multilayer shells. Biomaterials $\mathbf{3 0}$, 2799-2806 (2009).

107. Gu, Z. et al. Injectable nano-network for glucosemediated insulin delivery. ACS Nano 7, 4194-420 (2013).

108. Wu, W. \& Zhou, S. Responsive materials for selfregulated insulin delivery. Macromolec. Biosci. 13, 1464-1477 (2013).

109. Zion, T. C. Glucose-responsive materials for selfregulated insulin delivery. Thesis, Massachusetts Institute of Technology (2004).

110. Powell, A. E. \& Leon, M. A. Reversible interaction of human lymphocytes with the mitogen concanavalin A Exp. Cell Res. 62, 315-325 (1970)

111. Cartwright, H. A Review of deal making in 2010. 2011,17 PharmaDeals Review (2010)

112. Schechter, A. H. \& Perlmutter, R. M. in 2014 Merk Investor Briefing Webcast (ed. Frazier, K.) (Merck \& Co., 2014).

113. Matsumoto, A. et al. A synthetic approach toward self-regulated insulin delivery system. Angew. Chem. Int. Ed. Engl. 51, 2124-2128 (2012).

114. Wu, Q., Wang, L., Yu, H., Wang, J. \& Chen, Z. Organization of glucose-responsive systems and their properties. Chem. Rev. 111, 7855-7875 (2011).

115. Bae, Y., Fukushima, S., Harada, A. \& Kataoka, K. Design of environment-sensitive supramolecular assemblies for intracellular drug delivery: polymeric micelles that are responsive to intracellular $\mathrm{pH}$ change. Angewandte Chemie 42, 4640-4643 (2003).
116. Matsumoto, A., Yoshida, R. \& Kataoka, K Glucose-responsive polymer gel bearing phenylborate derivative as a glucose-sensing moiety operating at the physiological pH. Biomacromolecules 5, 1038-1045 (2004).

117. Uchiyama, T., Kiritoshi, Y., Watanabe, J. \& Ishihara, K. Degradation of phospholipid polymer hydrogel by hydrogen peroxide aiming at insulin release device. Biomaterials 24, 5183-5190 (2003).

118. Korin, N. et al. Shear-activated nanotherapeutics for drug targeting to obstructed blood vessels. Science 337, 738-742 (2012)

119. Yavuz, M.S. et al. Gold nanocages covered by smart polymers for controlled release with near-infrared light. Nature Mater. 8, 935-939 (2009).

120. Stanley, S. A. et al. Radio-wave heating of iron oxide nanoparticles can regulate plasma glucose in mice. Science 336, 604-608 (2012).

121. $\mathrm{Di}, \mathrm{J}$. et al. Ultrasound-triggered regulation of blood glucose levels using injectable nano-network. Adv. Healthc. Mater. 3, 811-816 (2014).

122. Bakhru, S. H., Furtado, S., Morello, A. P. \& Mathiowitz, E. Oral delivery of proteins by biodegradable nanoparticles. Adv. Drug Deliv. Rev. 65, 811-821 (2013)

123. Rabanel, J. M., Aoun, V., Elkin, I., Mokhtar, M \& Hildgen, P. Drug-loaded nanocarriers: passive targeting and crossing of biological barriers. Curr. Med. Chem. 19, 3070-3102 (2012).

124. Jin, Y. et al. Goblet cell-targeting nanoparticles for oral insulin delivery and the influence of mucus on insulin transport. Biomaterials 33, 1573-1582 (2012)

125. Sahay, G., Alakhova, D. Y. \& Kabanov, A. V. Endocytosis of nanomedicines. J. Control Release 145, 182-195 (2010)

126. Jepson, M. A., Clark, M. A. \& Hirst, B. H. M cell targeting by lectins: a strategy for mucosal vaccination and drug delivery. Adv. Drug Deliv. Rev. 56, 511-525 (2004).

127. Sweet, D. M., Kolhatkar, R. B., Ray, A., Swaan, P. \& Ghandehari, H. Transepithelial transport of PEGylated anionic poly(amidoamine) dendrimers: implications for oral drug delivery. J. Control Release 138, 78-85 (2009).

128. Pridgen, E. M. et al. Transepithelial transport of fc-targeted nanoparticles by the neonatal fc receptor for oral delivery. Sci. Transl. Med. 5, 213 ra167 (2013).

129. Edwards, D. A. et al. Large porous particles fo pulmonary drug delivery. Science 276, 1868-1871 (1997).

130. Patel, B., Gupta, V. \& Ahsan, F. PEG-PLGA based large porous particles for pulmonary delivery of a highly soluble drug, low molecular weight heparin. J. Control Release 162, 310-320 (2012).

131. Tang, B. C. et al. Biodegradable polymer nanoparticles that rapidly penetrate the human mucus barrier. Proc. Natl Acad. Sci. USA 106, 19268-19273 (2009).

132. Choi, H. S et al. Rapid translocation of nanoparticles from the lung airspaces to the body. Nature Biotech. 28, 1300-1303 (2010)

133. Liu, J. et al. Solid lipid nanoparticles for pulmonary delivery of insulin. Int. J. Pharmaceut. 356, 333-344 (2008)

134. Forst, T. et al. Time-action profile and patient assessment of inhaled insulin via the Exubera device in comparison with subcutaneously injected insulin aspart via the FlexPen device. Diabetes Technol. Ther 11, 87-92 (2009).

135. Hollander, P. A. et al. Efficacy and safety of inhaled insulin (Exubera) compared with subcutaneous insulin therapy in patients with type 2 diabetes: results of a 6 -month, randomized, comparative trial. Diabetes Care 27, 2356-2362 (2004)

Presents results from a clinical tria demonstrating safety and efficacy of the inhaled insulin formulation Exubera.

136. Mastrandrea, L. D. \& Quattrin, T. Clinical evaluation of inhaled insulin. Adv. Drug Deliv. Rev. 58 1061-1075 (2006)

137. Fischer, A. FDA approves Afrezza to treat diabetes. FDA News Release [online]

138. Neumiller, J. J. \& Campbell, R. K. Technosphere insulin: an inhaled prandial insulin product. BioDrugs 24, 165-172 (2010).

139. Prausnitz, M. R. \& Langer, R. Transdermal drug delivery. Nature Biotech. 26, 1261-1268 (2008)

140. Nose, K., Pissuwan, D., Goto, M., Katayama, Y. \& Niidome, T. Gold nanorods in an oil-base formulation for transdermal treatment of type 1 diabetes in mice. Nanoscale 4, 3776-3780 (2012).
141. Choi, W. I. et al. Efficient skin permeation of soluble proteins via flexible and functional nano-carrier. J. Control Release 157, 272-278 (2012).

142. Lopez, R. F., Seto, J. E., Blankschtein, D. \& Langer, R. Enhancing the transdermal delivery of rigid nanoparticles using the simultaneous application of ultrasound and sodium lauryl sulfate. Biomaterials 32, 933-941 (2011)

143. Higaki, M. et al. Transdermal delivery of $\mathrm{CaCO}_{3}$-nanoparticles containing insulin. Diabetes Technol. Ther. 8, 369-374 (2006).

144. Robertson, R. P. Islet transplantation as a treatment for diabetes, a work in progress. N. Engl. J. Med. 350, 694-705 (2004).

Excellent review providing a historical perspective on the challenges and opportunities for islet cell transplantation.

145. Gates, R. J., Hunt, M. I., Smith, R. \& Lazarus, N. R. Return to normal of blood-glucose, plasma-insulin, and weight gain in New Zealand obese mice after implantation of islets of Langerhans. Lancet 2 567-570 (1972).

\section{First demonstration of the concept of} immunoisolating pancreatic islet cells in semipermeable barriers.

146. Teramura, Y. \& Iwata, H. Bioartificial pancreas microencapsulation and conformal coating of isle of Langerhans. Adv. Drug Deliv. Rev. 62, 827-840 (2010)

147. Lanza, R. P., Hayes, J. L. \& Chick, W. L. Encapsulated cell technology. Nature Biotech. 14, 1107-1111 (1996).

148. Wilson, J. T., Cui, W. \& Chaikof, E. L. Layer-by-layer assembly of a conformal nanothin PEG coating for intraportal islet transplantation. Nano Lett. 8, 1940-1948 (2008)

149. Krol, S. et al. Multilayer nanoencapsulation. New approach for immune protection of human pancreatic islets. Nano Lett. 6, 1933-1939 (2006)

150. Contreras, J. L. et al. A novel approach to xenotransplantation combining surface engineering and genetic modification of isolated adult porcine islets. Surgery 136, 537-547 (2004).

151. Dolgin, E. Encapsulate this. Nature Med. 20, 9-11 (2014).

152. Buder, B., Alexander, M., Krishnan, R. Chapman, D. W. \& Lakey, J. R. Encapsulated islet transplantation: strategies and clinical trials. Immune Netw. 13, 235-239 (2013).

153. Czech, M. P., Aouadi, M. \& Tesz, G. J. RNAi-based therapeutic strategies for metabolic disease. Nature Rev. Endocrinol. 7, 473-484 (2011).

154. Li, F. \& Mahato, R. I. RNA interference for improving the outcome of islet transplantation. Adv. Drug Deliv. Rev. 63, 47-68 (2011).

155. Ko, K. S., Lee, M., Koh, J. J. \& Kim, S. W. Combined administration of plasmids encoding IL- 4 and IL-10 prevents the development of autoimmune diabetes in nonobese diabetic mice. Mol. Ther. 4, 313-316 (2001).

156. Oh, S., Lee, M., Ko, K. S., Choi, S. \& Kim, S. W. GLP-1 gene delivery for the treatment of type 2 diabetes. Mol. Ther. 7, 478-483 (2003)

157. Moon, J. J., Huang, B. \& Irvine, D. J. Engineering nano- and microparticles to tune immunity. Adv. Mater. 24, 3724-3746 (2012).

158. Nochi, T. et al. Nanogel antigenic protein-delivery system for adjuvant-free intranasal vaccines. Nature Mater. 9, 572-578 (2010).

159. Zhu, Q. et al. Large intestine-targeted, nanoparticlereleasing oral vaccine to control genitorectal viral infection. Nature Med. 18, 1291-1296 (2012).

160. Nembrini, C. et al. Nanoparticle conjugation of antigen enhances cytotoxic T-cell responses in pulmonary vaccination. Proc. Natl Acad. Sci. USA 108, E989-E997 (2011).

161. Kasturi, S. P. et al. Programming the magnitude and persistence of antibody responses with innate immunity. Nature 470, 543-547 (2011).

162. Geall, A. J. et al. Nonviral delivery of self-amplifying RNA vaccines. Proc. Natl Acad. Sci. USA 109, 14604-14609 (2012).

163. Nguyen, D. N. et al. Lipid-derived nanoparticles for immunostimulatory RNA adjuvant delivery. Proc. Nat Acad. Sci. USA 109, E797-E803 (2012).

164. Tsai, S. et al. Reversal of autoimmunity by boosting memory-like autoregulatory T cells. Immunity 32 568-580 (2010)

Notable study demonstrating the use of nanoparticles towards developing a diabetes vaccine. 
165. Hrkach, J. et al. Preclinical development and clinical translation of a PSMA-targeted docetaxel nanoparticle with a differentiated pharmacological profile. Sci. Transl. Med. 4, 128 ra39 (2012).

166. Davis, M. E. et al. Evidence of RNAi in humans from systemically administered siRNA via targeted nanoparticles. Nature 464, 1067-1070 (2010).

167. Peer, D. et al. Nanocarriers as an emerging platform for cancer therapy. Nature Nanotech. 2, 751-760 (2007).

168. Barenholz, Y. Doxil ${ }^{\circledR}$ - the first FDA-approved nano-drug: lessons learned. J. Control Release 160, 117-134 (2012). A notable review highlighting the development of the first FDA-approved nanoparticle-based therapeutic.

169. Bertrand, N., Wu, J., Xu, X., Kamaly, N. \& Farokhzad, O. C. Cancer nanotechnology: the impact of passive and active targeting in the era of modern cancer biology. Adv. Drug Deliv. Rev. 66, 2-25 (2014).

170. McCarthy, J. R. Nanomedicine and cardiovascular disease. Curr. Cardiovasc. Imag. Rep. 3, 42-49 (2010).

171. Lobatto, M. E., Fuster, V., Fayad, Z. A. \& Mulder, W. J. Perspectives and opportunities for nanomedicine in the management of atherosclerosis. Nature Rev. Drug Discov. 10, 835-852 (2011).
172. Alam, S. R. et al. Ultrasmall superparamagnetic particles of iron oxide in patients with acute myocardial infarction: early clinical experience. Circ. Cardiovasc. Imag. 5, 559-565 (2012).

173. Kim, B. Y. S., Rutka, J. T. \& Chan, W. C. W. Nanomedicine. New Engl. J. Med. 363, 2434-2443 (2010).

174. Ventura, J. FDA issues guidance to support the responsible development of nanotechnology products. FDA News Release [online] FDA guidelines to help foster the safe development of nanotechnology based products for clinical use.

175. Whitehead, K. A., Langer, R. \& Anderson, D. G. Knocking down barriers: advances in siRNA delivery. Nature Rev. Drug Discov. 8, 129-138 (2009).

176. Yi, P., Park, J. S. \& Melton, D. A. Betatrophin: a hormone that controls pancreatic $\beta$ cell proliferation Cell 153, 747-758 (2013).

177. Grunberger, G. The need for better insulin therapy Diabetes Obes. Metab. 15 (Suppl. 1), 1-5 (2013)

178. Keenan, D. B., Mastrototaro, J. J., Voskanyan, G. \& Steil, G. M. Delays in minimally invasive continuous glucose monitoring devices: a review of current technology. J. Diabetes Sci. Technol. 3, 1207-1214 (2009)
179. Hovorka, R. Closed-loop insulin delivery: from bench to clinical practice. Nature Rev. Endocrinol. 7 385-395 (2011)

180. Hovorka, R., Nodale, M., Haidar, A. \& Wilinska, M. E. Assessing performance of closed-loop insulin delivery systems by continuous glucose monitoring: drawbacks and way forward. Diabetes Technol. Ther. 15, 4-12 (2013).

181. Schmid, C., Haug, C., Heinemann, L. \& Freckmann, G. System accuracy of blood glucose monitoring systems: impact of use by patients and ambient conditions. Diabetes Technol. Ther. 15, 889-896 (2013).

Acknowledgements

We thank M. Anderson for helpful discussions and J. Gunn for his input towards the preparation of figure displays. Work in the author's laboratory was supported by the Leona M. and Harry B. Helmsley Charitable Trust Foundation (Grant 09PG-T1D027), the Juvenile Diabetes Research Foundation (JDRF) (Grant 17-2007-1063), the US National Institutes of Health (Grants EB000244, EB000351, DE013023 and CA151884) and a generous gift from the Tayebati Family Foundation. O.V. and B.C.T. were supported by the JDRF postdoctoral fellowships (Grants 3-2013-178 and 3-2011-310, respectively).

\section{Competing interests statement}

The authors declare competing interests: see Web version for details. 